\title{
A Juvenile Pleomorphic adenoma of the palate
}

\author{
Bouthaina Hammami ${ }^{1}$, Mohamed Amine Chaabouni ${ }^{1}$, Ghada Yousfi ${ }^{2}$, Omar Walha ${ }^{2}$, \\ Malek Mnejja ${ }^{3}$, Imen Achour ${ }^{3}$, and Ilhem Charfeddine ${ }^{1}$ \\ ${ }^{1}$ University of Sfax Faculty of Medecine of Sfax \\ ${ }^{2}$ Habib Bourguiba Hospital \\ ${ }^{3}$ Université de Sfax Faculté de Médecine de Sfax
}

September 29, 2021

\begin{abstract}
Salivary gland tumors in children are rare, involvement of accessory salivary glands is exceptional. We report a case of pleomorphic adenoma of the palate in a child in order to describe the clinical, radiological features, as well as the management of this rare localization.

\section{Introduction:}

Benign salivary tumors are rare in childhood; Only 3 to $5 \%$ of all salivary gland neoplasms occur in children and adolescents [1]. Pleomorphic adenomas (PAs) are the most common benign neoplasms in salivary glands. Between 70 and $85 \%$ of pleomorphic adenoma (PA)occur in the parotid gland, while $5 \%$ occur in the minor salivary glands [2]. Between minor salivary glands, the palate is the most commonly affected site, but other intraoral sites were reported as the upper and lower lips, buccal mucosa, gingival, and tongue [3,4]. We report a case of pleomorphic adenoma of the palate in a child to discuss the clinical features, treatment, and prognosis of this unusual localization.
\end{abstract}

\section{Case report:}

A 12-year- old girl, without a remarkable medical or surgical history, was referred to our department by her dentist for the discovery of a swelling of the palate. This mass was appeared two months below. No complaints were reported by the patient.

Clinical examination revealed a firm, nontender, nodular swelling over the left hard palate, measuring $1.5 * 1.5$ $\mathrm{cm}$, situated adjacent to the upper left second molar. No signs of inflammation or surface ulcer were noted (Figure 1)

Dental examination was normal. There were no neck lymph nodes.

A computed tomography (CT) scan of the massive facial, done with axial and coronal cuts, with contrast injection, showed a regular, limited tumor in the left soft palate, measuring $1.6 \mathrm{~cm}$, exerting a mass effect on the hard palate without perforation of the under lying bone (Figure 2).

Excision of the lump was carried out under general anesthesia. A palatal incision was raised from the upper left second premolar to the upper left second molar.

During the operation, the capsule was thin and discontinuous, the lump was excised with the capsule and the overlying mucosa. The defect was allowed to granulate (Figure 3)

The histopathologic examination of the specimen revealed a pleomorphic adenoma without signs of malignancy 
The patient was reviewed, two weeks postoperatively we noted good wound healing (Figure 4).

The child was followed regularly for 6 months and there was no sign of recurrence.

\section{Discussion}

Pleomorphic adenoma arising in the palate of children and adolescents is unusual. According to Ritwik, $28.6 \%$ of pleomorphic adenoma arising from minor salivary glands in children accrue over the first decade of life, with a female gender predilection of 2.8:1. The hard and/or soft palate is affected in $69.1 \%$ of cases [1].

Pleomorphic adenoma is usually a painless, slow-growing tumor; the duration of symptoms is variable, in regard to Honghai, it ranges from 20 days to 4 years [5].

In the most cases, palatal pleomorphic adenoma causes an asymptomatic, firm bulge in the palatal mucosa, although a few cases have exhibited ulceration and bleeding, usually resulting from trauma during mastication. It should be noted that PA tends to be small and fixed in palate as well as in the others minor salivary glands tumors compared to parotid where the lesion tends to be larger and mobile $[2,6,7]$. In our case the tumor was asymptomatic, with accidental discovery by a dentist.

In this localization, tumors are usually seen at the junction of the hard and soft palates [5].

For the size of the tumor, a study of 74 pleomorphic adenoma of the palate in adults and children showed an average size of $1.9 \mathrm{~cm}$, the tumors size ranged from 1 to $4 \mathrm{~cm}[8]$.

Magnetic resonance imaging and CT are recommended in tumors salivary glands, Computed tomography is more commonly used in the diagnosis of minor salivary gland PA. For palatal pleomorphic adenoma coronal view scans are recommended. They are used to determinate the size of palate lesions as well as verifying any bony involvement. But this technique exposes the child to ionizing radiation and it is limited in the characterization of soft tissue.

Nora reported a case of an incidental finding of palatal pleomorphic adenoma in the cone beam CT, due to pressure resorption of the hard palate, without break in the floor of the nasal cavity [7].

Magnetic resonance imaging provides excellent soft tissue characterization and does not expose patients to any radiation, but it can't evaluate the bone involvement in the palate localization [6].

Ultrasound has limited indication in palate lesion. For other minor salivary glands lesions, it is only used for buccal and parapharyngeal space masses [6].

Fine-needle aspiration can provide up to $80 \%$ accuracy in the diagnostic of salivary glands tumor, but young patients may not tolerate this procedure without sedation, making it a less favorable diagnostic tool than no invasive imaging techniques [3.5].

Surgical treatment of palatal pleomorphic adenoma in children is the same as in adults, it involves a wide local excision with its capsule and removal of the involved bone to avoid recurrence. According to Moon, In the cases Where the overlying mucosa is ulcerated or there was a capsule infiltration, or thin capsule in biopsy before surgery, the overlying mucosa should excised in order to prevent recurrence. the defect is either allowed to granulate or repaired with a local flap [9].

In our case the capsule was thin and discontinuous. Consequently, mucosal resection is performed with safety margin.

When substance loss is significant, fabricated palatal splints are sometimes inserted to reduce postoperative hematoma and ensure that the palatal flap is closely approximated to the palate [3]. Recurrence and carcinoma arising from PA are the main risk of pleomorphic adenomas [10]. According to a literature review published by Daniels, the recurrence rate is $12.5 \%$, In one case recurrence is observed after 5 years [3]. Moon reported one case of recurrence of carcinoma ex adenoma pleomorphic, the tumor was in capsular rupture during the operation [9]. Therefore, a complete resection with free limits, and prolonged as well as regular appropriate clinical monitoring are required, to improve the prognostic of these tumors. 


\section{Conclusion}

Pleomorphic adenoma of minor salivary glands is rare in pediatric population; palate is the most commune localization. Imaging, especially CT scan is important for planning surgical treatment. Complete resection with negative margins is the best option to avoid recurrence. Nevertheless, patients and doctors should remain vigilant for any recurrence that may occur in the follow-up period which must be extended to five years at least.

\section{ACKNOWLEDGMENTS}

All authors: Approved the final version and have the agreement to be accountable for all aspects of the work in ensuring that questions related to the accura.

\section{CONFLICT OF INTEREST}

None declared.

\section{AUTHOR CONTRIBUTIONS}

GY and MAC: wrote the manuscript with support of OW. BH, IA,. MM and IC: approved the final version of the manuscript.

\section{ETHICS APPROVAL AND CONSENT}

Obtained from parents of the child in written.

\section{DATA AVAILABILITY STATEMENT}

The datasets used and/or analyzed during the current study are available from the corresponding author on reasonable request.

\section{References}

1. Ritwik P, Brannon RB. A clinical analysis of nine new pediatric and adolescent cases of benign minor salivary gland neoplasms and a review of the literature. J Med Case Rep. 2012;6:287.

2. Shaaban H, Bruce J, Davenport PJ. Recurrent pleomorphic adenoma of the palate in a child. British Joumal of Plastic Surgery; 245-247

3. Daniels JSM, Ali I, Al Bakri IM, Sumangala B. Pleomorphic adenoma of the palate in children and adolescents: a report of 2 cases and review of the literature. J Oral Maxillofac Surg. 2007;65(3):541-9.

4. Dhanuthai K, Sappayatosok K, Kongin K. Pleomorphic adenoma of the palate in a child: a case report. Med Oral Patol Oral Cir Bucal. 2009;14(2):73-75.

5. Fu H, Wang J, Wang L, Zhang Z, He Y. Pleomorphic adenoma of the salivary glands in children and adolescents. J Pediatr Surg. 2012;47(4):715-9.

6. Dombrowski ND, Wolter NE, Irace AL, Cunningham MJ, Vargas SO, Perez-Atayde AR, et al. Pleomorphic adenoma of the head and neck in children: presentation and management. Laryngoscope. 2019;129(11):2603-9.

7. Alsufyani N. Incidental Cone Beam CT Finding of Juvenile Pleomorphic Adenoma Case Reports in Dentistry. Hindawi; 2020 p. e8862657.

8. Wu Y-C, Wang Y-P, Cheng S-J, Chen H-M, Sun A, Chang JY-F. Clinicopathological study of 74 palatal pleomorphic adenomas. J Formos Med Assoc. 2016;115(1):25-30.

9. Moon S-Y. Surgical Management of the Palatal Pleomorphic Adenoma. J Craniofac Surg. 2019;30(6):580-2.

10. Pramod Krishna B. Pleomorphic Adenoma of Minor Salivary Gland in a 14 year Old Child. J Maxillofac Oral Surg. 2013;12(2):228-31. 


$$
\text { 望 }
$$




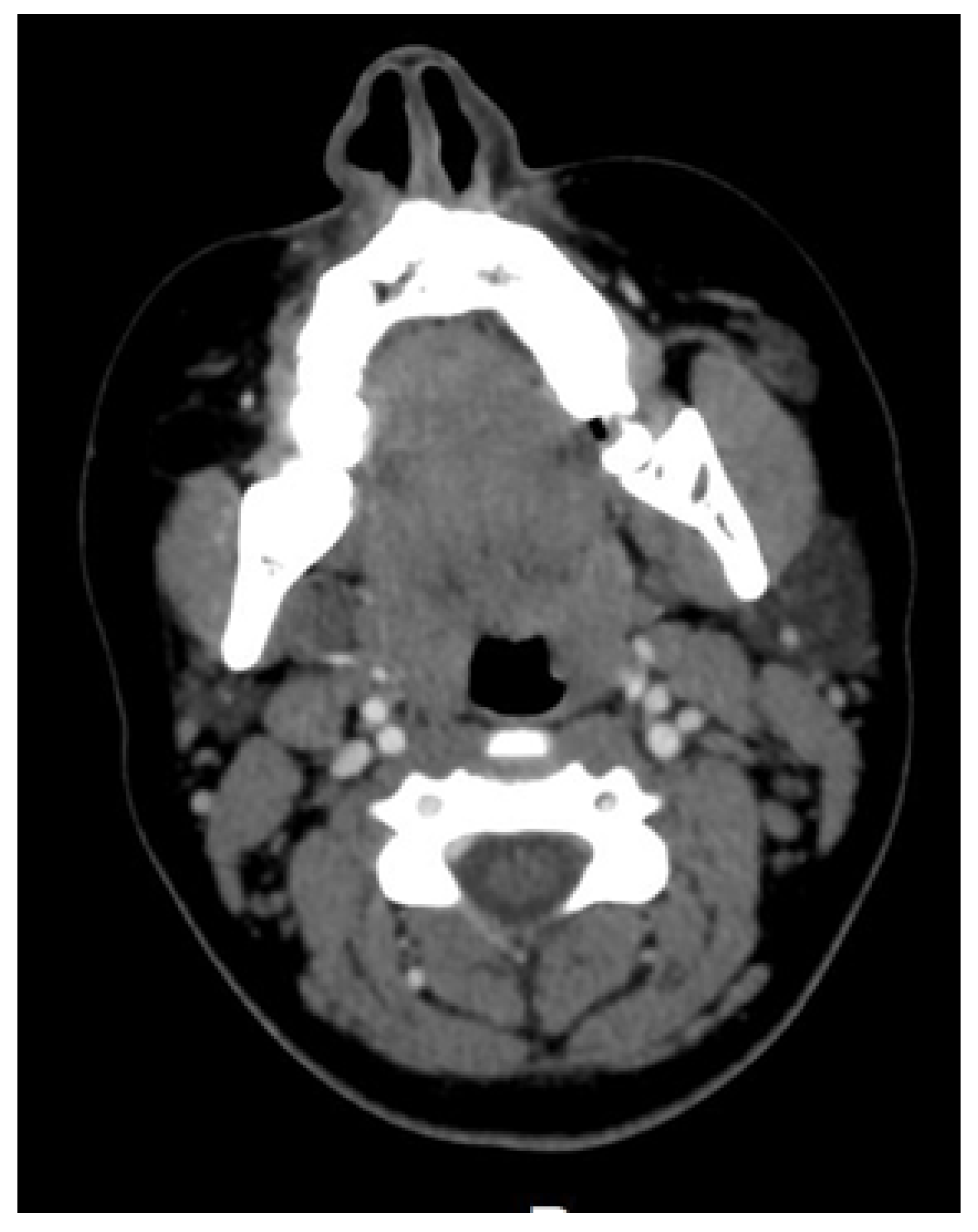




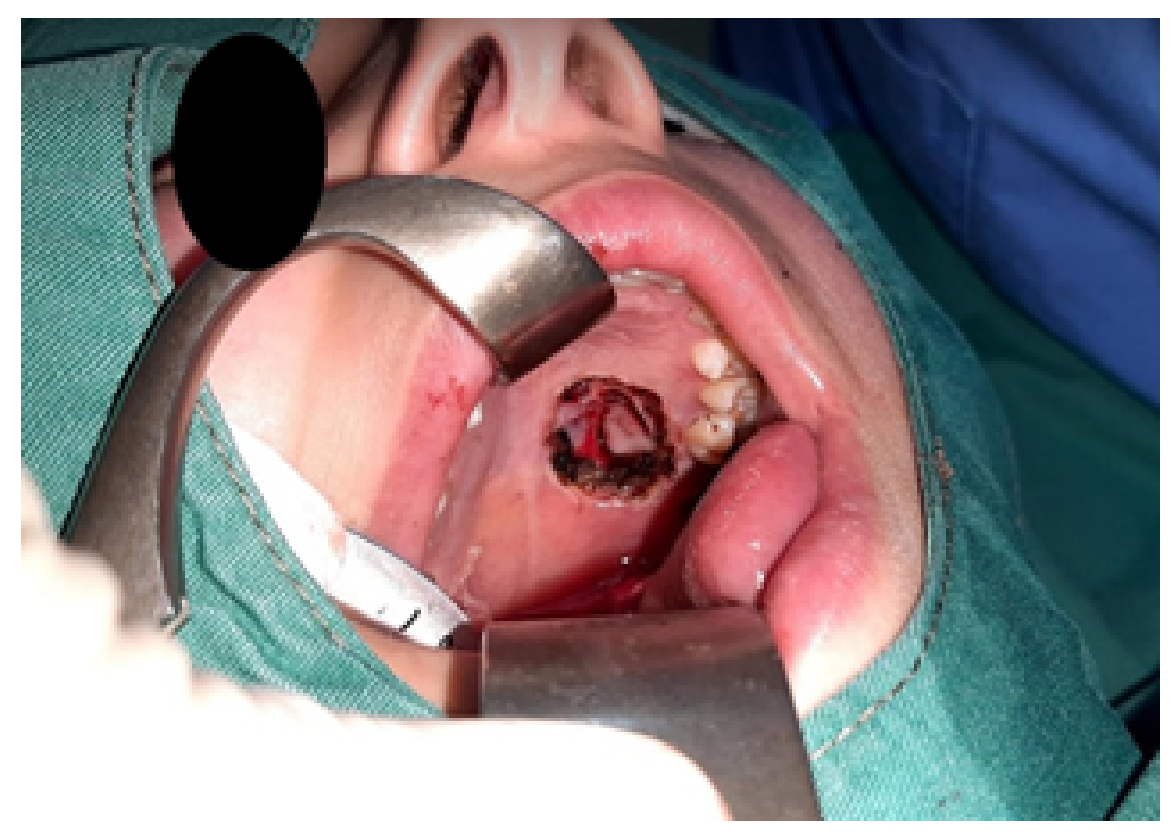




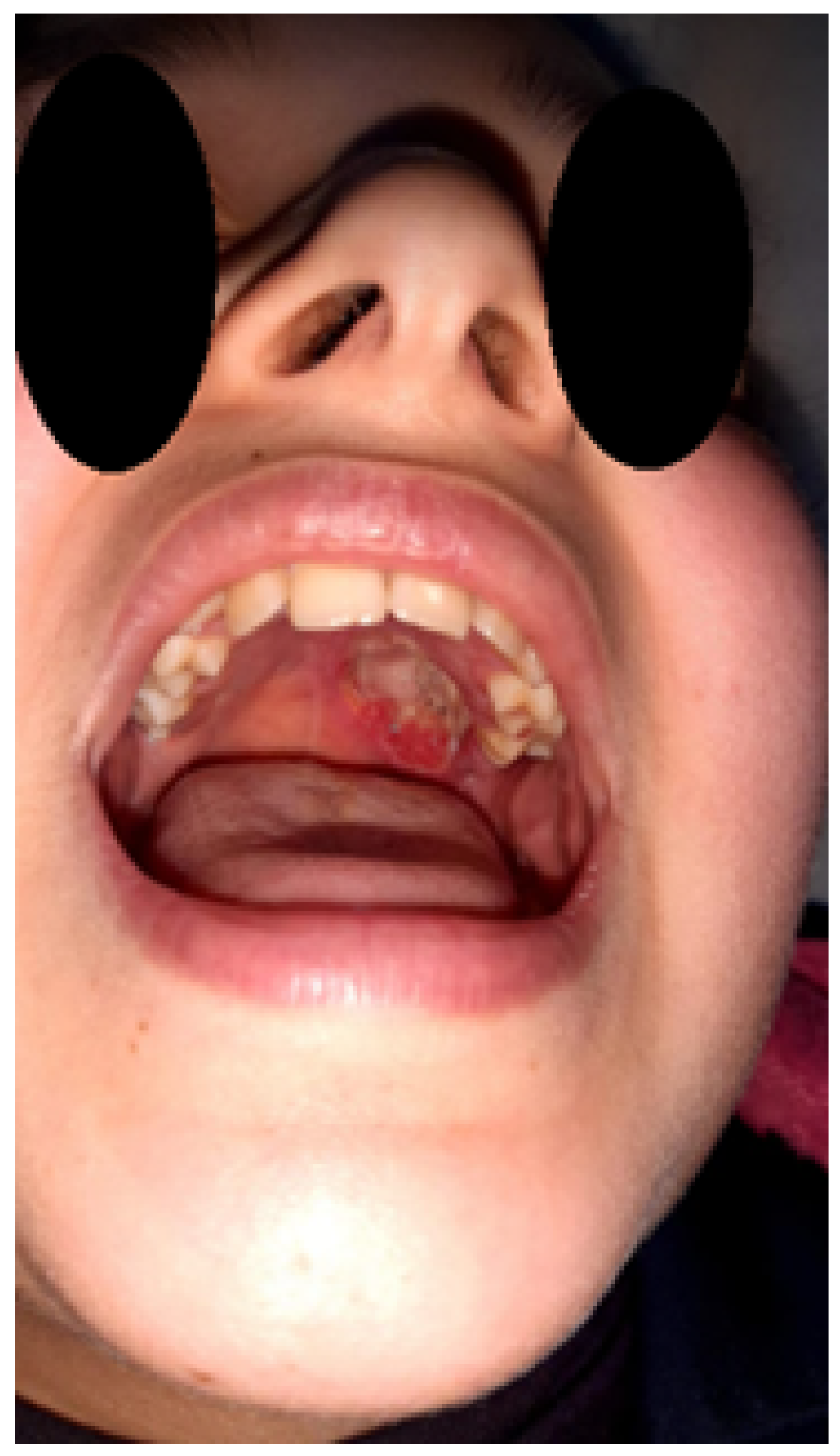

